\title{
Chromosomal mapping of pancreatic islet morphological features and regulatory hormones in the spontaneously diabetic (Type 2) Goto-Kakizaki rat
}

\author{
Clare Finlay · Karène Argoud · Steven P. Wilder • \\ Fetta Ouali • Alain Ktorza • Pamela J. Kaisaki • \\ Dominique Gauguier
}

Received: 4 July 2010/ Accepted: 1 September 2010/Published online: 29 September 2010

(C) The Author(s) 2010. This article is published with open access at Springerlink.com

\begin{abstract}
Insulin resistance and altered endocrine pancreas function are central pathophysiological features of type 2 diabetes mellitus (T2DM). The Goto-Kakizaki (GK) rat is a model of spontaneous T2DM characterised by reduced beta cell mass and genetically determined glucose intolerance and altered insulin secretion. To identify genetic determinants of endocrine pancreas histopathology, we carried out quantitative trait locus (QTL) mapping of histological phenotypes (beta cell mass -BCM and insulin-positive cell area -IPCA) and plasma concentration of hormones and growth factors in a F2 cohort derived from GK and normoglycemic Brown Norway rats. Although IPCA and BCM in the duodenal region of the pancreas were highly positively correlated $\left(P<10^{-6}\right)$, and similarly in the splenic region, both measures were poorly correlated when comparing duodenal and splenic phenotypes. Strongest evidence of linkage to
\end{abstract}

Electronic supplementary material The online version of this article (doi:10.1007/s00335-010-9285-3) contains supplementary material, which is available to authorized users.

C. Finlay $\cdot$ K. Argoud · S. P. Wilder · P. J. Kaisaki ·

D. Gauguier $(\bowtie)$

The Wellcome Trust Centre for Human Genetics, University of Oxford, Roosevelt Drive, Headington, Oxford OX3 7BN, UK

e-mail: gdomi@well.ox.ac.uk

F. Ouali

Laboratoire de Physiopathologie de la Nutrition,

CNRS UMR 7059 Paris, France

\section{A. Ktorza}

Institut de Recherches Servier, 92150 Suresnes, France

D. Gauguier

INSERM, U872, Centre de Recherche des Cordeliers,

Paris, France pancreas morphological traits was obtained between BCM and chromosome 10 (LOD 3.2). Evidence of significant linkage (LOD 4.2) to plasma corticosterone was detected in a region of chromosome 1 distal to other QTLs previously identified in the GK. Male-specific genetic effects were detected, including linkages (LOD $>4$ ) to growth hormome (GH) on chromosome 6 and prolactin on chromosome 17 . These data suggest independent genetic control of the structure and function of ontologically different regions of the endocrine pancreas. Novel QTLs for corticosterone, prolactin and GH may contribute to diabetes in the GK. The QTLs that we have identified in this, and previous genetic studies collectively underline the complex and multiple mechanisms involved in diabetes in the GK strain.

\section{Introduction}

Pancreatic islet anomalies and reduced sensitivity of peripheral tissues to insulin action (insulin resistance) are central features in the pathogenesis of type 2 diabetes (T2D) (Kahn 2003). Owing to the widely accepted existence of decreased beta cell mass and function in T2D (Kahn et al. 2009), there is growing interest in understanding structural and functional changes in pancreatic islets in diabetes and their relationships with insulin resistance. The endocrine part of the pancreas, which produces insulin, glucagon somatostatin and the pancreatic polypeptide, represents only $10 \%$ of the pancreas. It undergoes complex in utero and postnatal developmental maturation and functional processes involving intrinsic and circulating signalling factors (Habener et al. 2005; Puri and Hebrok 2010). Another level of complexity lies in the organogenesis of the pancreas which differentiates from two separate buds (ventral and dorsal) of cells of the gut 
endodermal epithelium, leading to the development and fusion of two primordial organs (Edlund 2001). This developmental regionalisation of the endocrine pancreas has long term structural and functional consequences on islet density and beta cell mass content in the corresponding regions of adult pancreas (Rahier et al. 2008; Spooner et al. 1970).

For obvious ethical reasons the regulation of structural and functional pancreatic phenotypes are difficult or impossible to investigate directly in diabetic patients and healthy individuals. Animal models of diabetes mellitus are crucially important experimental systems to investigate islet structural and functional variables and their genetic control. The inbred Goto-Kakizaki (GK) rat strain is a model of spontaneous diabetes, which develops as a consequence of the expression of naturally occurring disease gene variants isolated from an outbred Wistar colony. Even though the GK strain was derived following repeated breeding of rats selected solely on the basis of glucose intolerance (Goto et al. 1988) it exhibits a wide range of additional anomalies, including altered insulin secretion in vivo and in vitro, insulin resistance and reduced beta-cell mass (Portha 2005). Genetic studies in this model have primarily focused on the identification of genetic loci (quantitative trait loci-QTLs) involved in the regulation of diabetes and obesity intermediate physiological phenotypes (glucose tolerance, insulin secretion, body weight, adiposity, lipoprotein metabolism) (Argoud et al. 2006; Gauguier et al. 1996), and ${ }^{1} \mathrm{H}$ NMR metabonomic-derived metabolic variables (Dumas et al. 2007).

In the present work, we investigated the genetic control of phenotypes directly or indirectly relevant to beta cell structure and function in adult rats of an experimental cross derived from the GK and normoglycemic Brown Norway (BN) strains. We identified QTLs linked to beta cell mass and plasma concentration of corticosterone, prolactin and growth hormone. Results from this, and previous genetic studies, provide a comprehensive mapping of naturally occurring genetic variants that cause diabetes in the GK and further emphasise the complex etiology of diabetes in this model.

\section{Methods}

Animals

$\mathrm{BN} / \mathrm{Crl}$ rats were obtained from a commercial supplier (Charles River Laboratories, Margate, UK). Rats of the GK/Ox colony were bred locally. All rats were maintained on a 12-h light and dark cycle and had free access to water and standard laboratory chow pellets (ERB, Whitam, UK).
All procedures were carried out in accordance with national and institutional guidelines.

Two reciprocal $\mathrm{F} 1$ generations (female $\mathrm{GK} \times$ male $\mathrm{BN}$ and female $\mathrm{BN} \times$ male $\mathrm{GK}$ ) were produced. A cohort of $170 \mathrm{~F} 2$ rats ( 84 males and 86 females) was independently derived from F1 animals, 88 originated from a GK female and 82 from a GK male. Progenies were weaned at 21 days. At 2 months of age, rats were fasted overnight for 16-18 $\mathrm{h}$ and blood samples were collected at about 9am into EDTA-tubes. Plasma was separated by centrifugation at 3,000 RPM and immediately stored at $-80^{\circ} \mathrm{C}$ until assays. Rats were then killed by decapitation. The spleen of F2 hybrids was dissected and stored at $-80^{\circ} \mathrm{C}$ for DNA preparation. The pancreas of each hybrid was dissected and weighed prior to immunocytochemistry and morphometry studies.

Pancreatic islet immunocytochemistry and morphometry

Islet morphology was evaluated by immunostaining of pancreatic sections for insulin. The duodenal and splenic pancreatic regions were dissected, independently weighed, fixed in aqueous Bouin's solution and embedded in Paraplast (Labonord, France). Paraffin pancreatic blocks were serially sectioned $(7 \mu \mathrm{m})$ throughout the organ length to avoid possible bias caused by regional changes in islet distribution or cell composition, and mounted on slides. Seven sections were chosen at a fixed interval through the pancreatic block (every 35 th section) to provide a representation of the structure of the islets across whole pancreas. Paraffin sections were deparaffinized, rehydrated and immunostained for insulin using a peroxidase indirectlabelling technique.

Sections were incubated for $1 \mathrm{~h}$ with a guinea pig antiinsulin serum (final dilution 1:1,000; ICN, Aurora, OH) and incubated for $45 \mathrm{~min}$ with peroxidase-conjugated rabbit anti-guinea pig IgG (final dilution 1:20; Dako, Carpinteria, CA). The activity of the antibody-peroxidase complex was revealed with DAB (Diaminobenzidine-tetrahydrochloride) using a peroxidase substrate kit DAB (Biosys-Vector, Compiègne, France). A standard concentration of hematoxylin was used as a counterstain. After staining, sections were mounted in Eukitt (Kindler, Freiburg, Germany).

Quantitative measurements of islet structural phenotypes were performed using an Olympus $\mathrm{BH} 2$ microscope connected via a colour video camera to a computer, using Imagenia 2000 software (Biocom, Les Ulis, France). Area of insulin-positive cells and that of total pancreatic sections were measured in each section. Beta cell area was then determined by calculating the ratio between the areas according to stereological methods (Kargar and Ktorza 
2008). Finally, total beta-cell mass for the ventral and dorsal parts of the pancreas of each F2 hybrid was derived by multiplying this ratio by the weight of the pancreatic region.

Quantitative analysis of beta cell size was performed using a computer-assisted image analysis procedure based on an Olympus BX 40 microscope (Olympus-France, France) connected via video camera to a personal computer and using Visiolab 1000 software (Biocom, France). Beta cell size was measured by a stereological morphometric method which calculates on insulin-stained sections the mean cross-sectional area of individual beta-cells. Beta cell nuclei were counted on a random section, and the beta cell area in that section was measured by planimetry. Beta cell area was divided by the number of nuclei to calculate the area of individual beta cells.

\section{Analytical methods}

Plasma concentration of glucose was determined using commercial diagnostic kits on a Cobas Mira Plus automatic analyser (ABX, Shefford, UK) and plasma corticosterone was determined with a rat corticosterone EIA kit (DSL, Webster, Tx). Plasma growth hormone level was measured with a Mouse/Rat GH ELISA kit (DSL, Webster, Tx). Plasma prolactin concentration was determined with a rat prolactin ELISA kit (MD Biosciences Inc, MN). Concentrations of total and free insulin-like growth factor-I were determined by immunoenzymometric assay using a Rat/ Mouse IGF-1 IEMA kit (GroPep-IDS, Fountain Hills, USA). Bound IGF-I, free/total IGF-I and bound/total IGF-I were calculated from the total and free measured values. Immunoreactive insulin (IRI) was measured with an ELISA kit (Mercodia, Uppsala, Sweden). All assays were performed according to the manufacturer's instructions.

Genetic markers and genotype determination

Genomic DNA of the F2 rats was prepared from spleens as previously described (Gauguier et al. 1996). Rat microsatellite markers were selected in our linkage maps constructed in a GKxBN cross (Wilder et al. 2004) in order to provide a genome coverage of $10-15 \mathrm{cM}$ between adjacent loci on average in the new GKxBN F2 cross. Marker information is available at http://www.well.ox.ac.uk/ rat_mapping_resources. Genotypes of the F2 rats were determined using a PCR protocol previously described (Bihoreau et al. 1997). PCR products were separated by electrophoresis on $4 \%$ agarose gels and visualized by ethidium bromide gel staining for genotype determination. Oligonucleotides were synthesized by Sigma-Genosys (Pampisford, UK).
Construction of the genetic maps

Genetic maps were constructed using the JoinMap version 2.0 suite of programs (Stam 1993) as previously described (Bihoreau et al. 1997). Genotypes were verified prior to QTL analysis using JoinMap modules, which calculate for all loci and for all individuals the probability of obtaining the present genotype, conditional on both map distances and genotypes at the two flanking loci.

Statistical methods and QTL analysis

The R (Ihaka and Gentleman 1996) package SMA was used for testing phenotype correlations. All correlations between standardised residuals were calculated and plotted and their significance were adjusted for the multiple testing using the Dunn-Šidák method (Ury 1976). All phenotypes were corrected for sex by regression prior to QTL mapping and the standardized residuals were analyzed for departures from normality using the Kolmogorov-Smirnov and Shapiro-Wilk tests (SPSS version 12.0 package, SAS Institute Inc. Carry, NC). For those variables with significant departure from normality, the logarithm-transformed raw data were regressed by sex, and the standardised residuals were analysed for departures from normality. All further linkage analyses were carried out with the standardized residuals which best fitted the normal distribution. Linkages between marker genotypes and phenotypes were initially evaluated using automated ANOVA tests followed by permutation tests $(n=10,000)$, which were applied to calculate the threshold of significance for each pair of genetic-phenotypic marker (Doerge and Churchill 1996). The genome wide scans were applied using R/qtl (Broman et al. 2003). Haley-Knott regression (Haley and Knott 1992) was used for each of the standardized variables. Subsequently, genome scans were generated for the two sexes to test the existence gender effects in QTL detection. Finally, we used a two-sample $t$-test to test whether there were significant differences in standardised phenotypes between the two cross directions. Correlations between marker genotypes and phenotypes were calculated using the SPSS version 16.0 package (SAS Institute Inc. Carry, $\mathrm{NC})$.

\section{Results}

Phenotype investigations in GK and BN strains

To investigate islet architecture in young adult rats of the strains used to derive the GKxBN F2 cohort, pancreas histology was carried out in 2 month-old GK and BN rats. Typical morphological features of GK pancreas include 
reduction in insulin-positive cell area (IPCA) (Table 1, Fig. 1a, b) and fragmented appearance and compromised architecture of islets (Fig. 1c, d), when compared to BN. Reduction in IPCA in GK was significant in both duodenal and splenic pancreatic segments in males and in the splenic region in females (Table 1). Accordingly, beta cell mass (BCM) in both duodenal and splenic segments was significantly reduced in both male and female GK rats when compared to sex-matched BN rats. Body weight was significantly lower in BN rats than in sex matched GK.

To document possible causes and/or effects of altered islet morphology, the concentration of glucose, insulin and hormones known to play a role in or to be influenced by pancreas function were measured in plasma of 2 month-old GK and BN rats (Table 1). Male and female GK rats showed significant basal hyperglycemia and hyperinsulinemia when compared to BN. Reduced concentration of plasma prolactin and increased concentration of plasma corticosterone were observed in male GK when compared to sex matched BN.

Islet morphological and plasma hormone variables in $\mathrm{F} 2(\mathrm{GK} \times \mathrm{BN})$ hybrids

To evaluate the inheritance of these phenotypic features in hybrids of a cross between GK and BN rats, we initially analysed their distribution in F2 hybrids. Pancreata of F2 animals generally displayed levels of IPCA and BCM that spanned the interval between and extended beyond the parental strain means but with means intermediate between the parental strains (Table 1, Supplementary Fig. S1), with the exception of female duodenal IPCA and BCM, which were lower on average than the GK mean. Female F2 rats had significantly reduced mean IPCA and BCM when compared to male $\mathrm{F} 2$ rats in both duodenal and splenic segments of the pancreas (Table 1).

Mean glucose, insulin and corticosterone concentrations in F2 males and females were generally intermediate between parental means (Table 1). There was a decrease in the mean of GH and an increase in the means of IGF-1 and prolactin in $\mathrm{F} 2$ rats when compared to parental strains (Table 1, Supplementary Fig. S2). Female F2 rats showed significantly lower insulin and IGF-1 than males. As expected they also showed higher prolactin than F2 males.

Correlations between phenotypes quantified in $\mathrm{F} 2(\mathrm{GK} \times \mathrm{BN})$ hybrids

IGF-1, corticosterone, prolactin and glycemia showed no significant correlation with any of the other phenotypes in the study suggesting independent genetic control (Fig. 2). Growth hormone concentration was significantly positively associated with IPCA and BCM in the duodenal pancreas and accordingly with the total IPCA and BCM but the effect was not significant in the splenic pancreas. Conversely insulin has a trend towards a positive correlation with splenic pancreas phenotypes, significantly increasing the total BCM. Body weight was significantly correlated with both duodenal and splenic pancreas weights and $\mathrm{BCM}$, after adjusting for multiple testing. It was also significantly correlated with insulin concentration.

Table 1 Islet histopathological phenotypes, body weight and plasma concentrations of glucose, insulin, growth hormone (GH), insulin growth factor 1 (IGF-1), corticosterone and prolactin quantified in BN, GK and (GKxBN) F2 male and female rats

\begin{tabular}{|c|c|c|c|c|c|c|}
\hline & \multicolumn{2}{|l|}{$\mathrm{BN}$} & \multicolumn{2}{|l|}{ GK } & \multicolumn{2}{|l|}{$(\mathrm{GKxBN}) \mathrm{F} 2$} \\
\hline & Males (6) & Females (6) & Males (6) & Females (6) & Males (84) & Females (86) \\
\hline IPCA (duodenal) (\%) & $0.67 \pm 0.07$ & $0.56 \pm 0.12$ & $0.34 \pm 0.06^{*}$ & $0.39 \pm 0.06$ & $0.45 \pm 0.02$ & $0.35 \pm 0.01 \uparrow \dagger \dagger$ \\
\hline IPCA (splenic) $(\%)$ & $0.75 \pm 0.13$ & $0.80 \pm 0.10$ & $0.34 \pm 0.05^{*}$ & $0.34 \pm 0.04 * *$ & $0.57 \pm 0.02$ & $0.51 \pm 0.02 \uparrow \dagger \dagger$ \\
\hline BCM (duodenal) (mg) & $1.70 \pm 0.12$ & $1.38 \pm 0.10$ & $0.87 \pm 0.20^{*}$ & $0.95 \pm 0.08 *$ & $1.10 \pm 0.05$ & $0.74 \pm 0.03+\dagger \dagger$ \\
\hline BCM (splenic) (mg) & $3.75 \pm 0.28$ & $3.59 \pm 0.21$ & $1.08 \pm 0.17 * * *$ & $1.22 \pm 0.26^{* * *}$ & $2.53 \pm 0.11$ & $1.91 \pm 0.08 \dagger \dagger \dagger$ \\
\hline BCM (total) (mg) & $5.39 \pm 0.30$ & $4.90 \pm 0.19$ & $2.00 \pm 0.40^{* * *}$ & $2.18 \pm 0.33^{* * *}$ & $3.63 \pm 0.13$ & $2.65 \pm 0.09$ \\
\hline Body weight (g) & $191 \pm 4$ & $155 \pm 6 \dagger \dagger$ & $224 \pm 7 * * *$ & $176 \pm 5^{* \dagger \dagger}$ & $216 \pm 3$ & $147 \pm 2 \dagger \dagger \dagger$ \\
\hline Plasma glucose (mM) & $7.10 \pm 0.17$ & $7.22 \pm 0.22$ & $14.37 \pm 1.44^{* * *}$ & $14.60 \pm 0.89 * * *$ & $11.54 \pm 0.17$ & $11.16 \pm 0.22$ \\
\hline Insulin $(\mathrm{pg} / \mathrm{ml})$ & $246 \pm 43$ & $233 \pm 31$ & $723 \pm 92^{* *}$ & $350 \pm 53^{*} \dagger$ & $398 \pm 17$ & $300 \pm 15 \dagger \dagger \dagger$ \\
\hline $\mathrm{GH}(\mathrm{ng} / \mathrm{ml})$ & $59 \pm 10$ & $101 \pm 5 \dagger \dagger$ & $66 \pm 3$ & $66 \pm 23$ & $24 \pm 2$ & $20 \pm 2$ \\
\hline IGF-1 (ng/ml) & $846 \pm 288$ & $665 \pm 48 \dagger$ & $1180 \pm 162$ & $849 \pm 76 \dagger$ & $1930 \pm 59$ & $1750 \pm 64 \dagger$ \\
\hline Corticosterone (ng/ml) & $283 \pm 30$ & $441 \pm 67$ & $523 \pm 86^{*}$ & $604 \pm 65$ & $401 \pm 25$ & $380 \pm 29$ \\
\hline Prolactin (ng/ml) & $36 \pm 5$ & $113 \pm 17 \dagger \dagger$ & $7 \pm 2 * *$ & $133 \pm 33 \dagger \dagger$ & $62 \pm 8$ & $144 \pm 10 \dagger \dagger \dagger$ \\
\hline
\end{tabular}

Beta cell mass (BCM) and insulin-positive cell area (IPCA) were quantified in the duodenal and splenic regions of the pancreas. Values are means \pm SE. Number of rats is in parentheses. $* P<0.05$, ** $P<0.01$, *** $P<0.001$ significantly different between GK and sex matched BN rats. $\dagger P<0.05$; $\dagger$ † $P<0.01 ; \dagger \dagger \uparrow P<0.001$ significant sex differences within groups 
Fig. 1 Pancreas histology in rats of the inbred $\mathrm{BN}(\mathbf{a}, \mathbf{c})$ and GK (b, d) strains. Pancreas sections $(7 \mu \mathrm{m})$ stained for insulin show the overall reduction in insulin-positive cell area in GK (b) rats compared to BN (a). Further magnification shows fragmented appearance and compromised architecture of the GK islets (d) when compared to $\mathrm{BN}(\mathbf{c})$

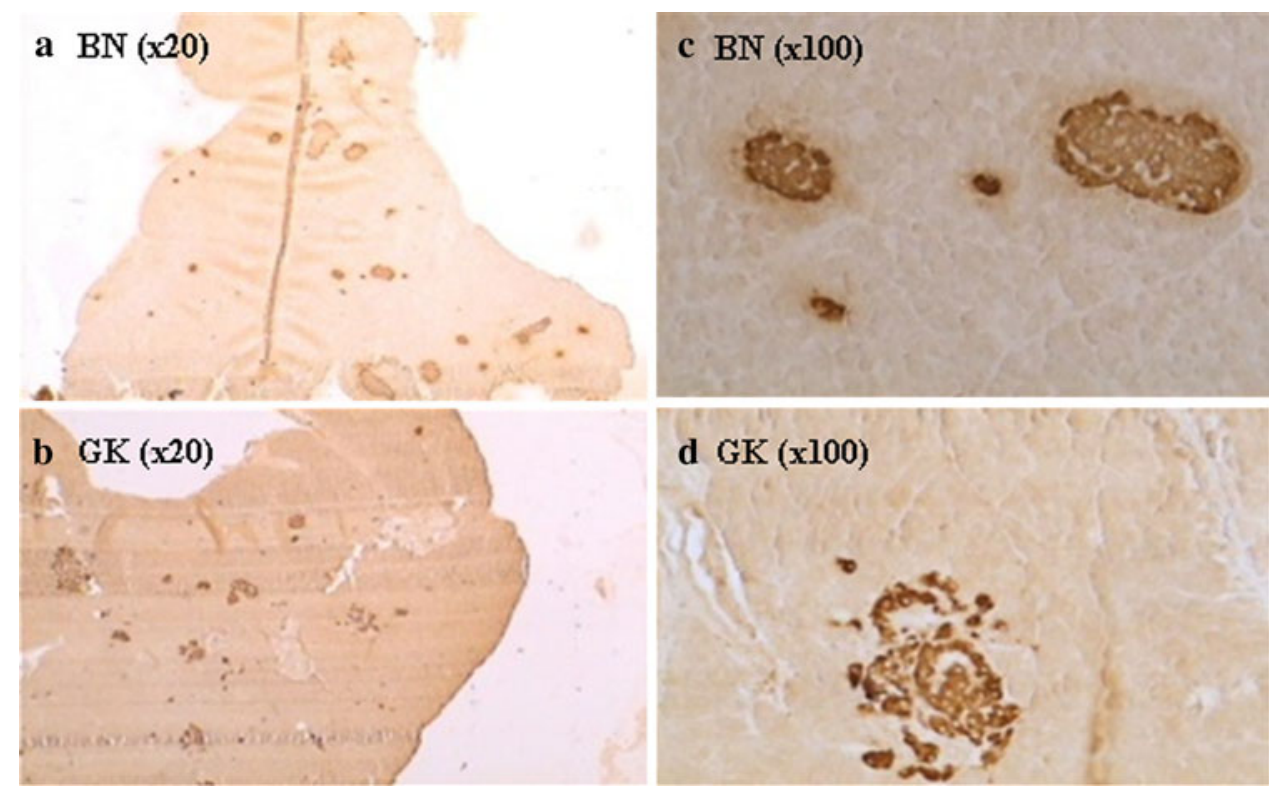

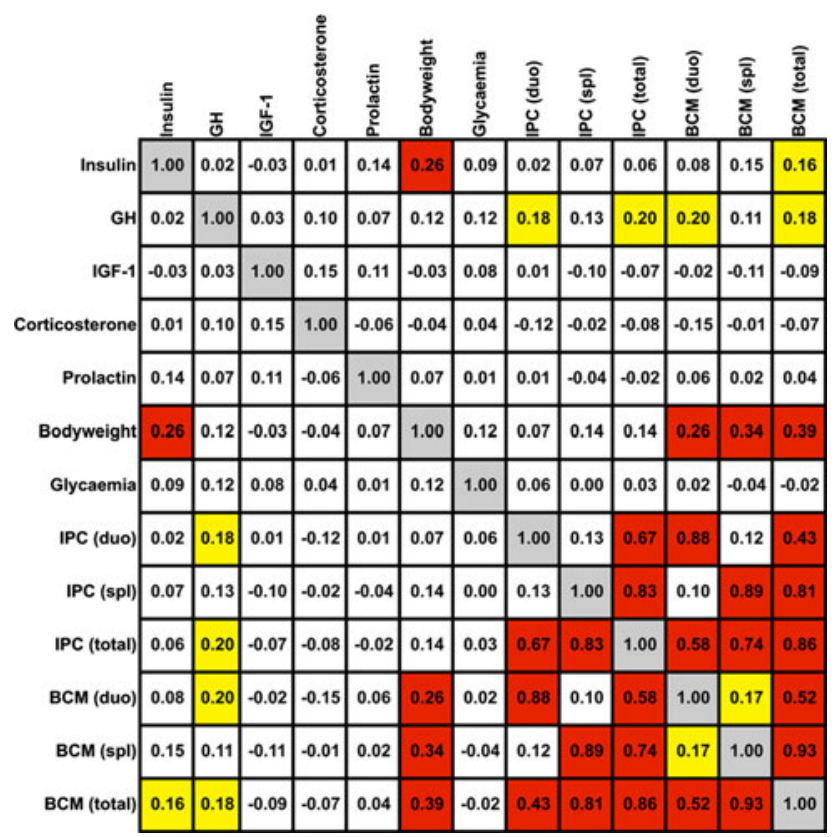

Fig. 2 Pearson correlations between phenotypes quantified in the GK $\times$ BN F2 cross (see Table 1). Each comparison is based on phenotypes regressed for sex effects acquired in over 143 F2 hybrids. Statistically significant (2-tailed) correlations are shown on yellow $(P<0.05)$ or red $(P<0.001)$ backgrounds. Duo duodenal segment, spl splenic segment, $B C M$ Beta cell mass, IPC insulin-positive cells

Correlation between splenic and duodenal BCM was marginally significant (Fig. 2). Interestingly, duodenal IPCA was highly positively correlated with duodenal BCM, and similarly in the splenic segment, but IPCA was poorly correlated when comparing between segments, suggesting independent genetic control of duodenal and splenic phenotypes in accordance with distinct organogenesis of these pancreatic regions.

Genetic map

A genetic map was constructed using genotype data generated from the 150 markers typed in GKxBN F2 hybrids. The markers spanned a total distance of $1620 \mathrm{cM}$ (Kosambi), which is consistent with our previously published linkage maps constructed in a similar F2 cross (Wilder et al. 2004). As expected the average distance between adjacent loci was $10.8 \mathrm{cM}$ and marker order was conserved with our previously published maps (Argoud et al. 2006; Wilder et al. 2004).

\section{Quantitative trait locus mapping}

To identify genetic loci controlling islet histopathological phenotypes and plasma hormones, genotype data from the genome wide scan carried out in the cross were used to test for linkage to quantitative phenotypes. Strongest evidence of linkage to BCM and IPCA in the splenic pancreatic region was detected with a locus in chromosome 10 (maximum LOD 3.2 at marker locus D10Got156) (Fig. 3a and Fig. 3b). This locus was denoted $\mathrm{Bcml}$. Interestingly, this locus does not show significant linkage to IPCA and $\mathrm{BCM}$ in the duodenal region (Fig. $3 \mathrm{c}$ and d), thus confirming the independent genetic control of splenic and duodenal pancreatic phenotypes suggested by the analysis of correlations. In the cross, GK genotypes at marker locus D10Got156 are associated with a significant reduction in IPCA (Fig. 3e) and BCM (Fig. 3f) in the splenic pancreas, but no significant differences in either measure in the 
$\mathrm{F}$
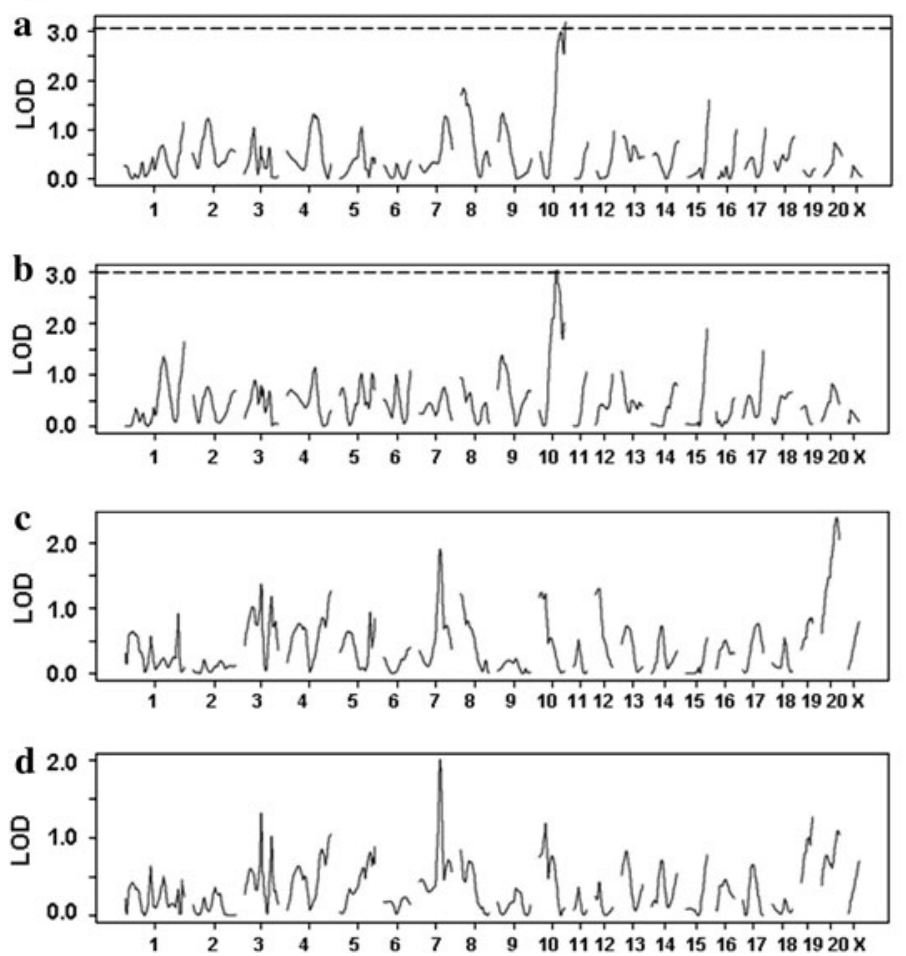
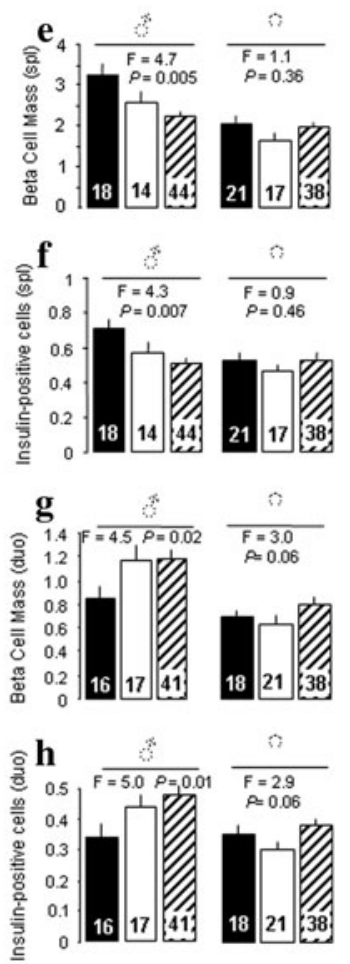

Fig. 3 Genome-wide genetic linkage analysis for beta cell mass $(\mathrm{BCM})(\mathbf{a}, \mathbf{c})$ and insulin-positive cell area (IPCA) $(\mathbf{b}, \mathbf{d})$ in the splenic (a, b) and duodenal (c, d) regions of the pancreas in the GK $\times$ BN F2 cross. LOD scores are plotted against map distance calculated in the cross in centimorgans $(\mathrm{cM})$. Dotted lines indicate statistically significant threshold of linkages $(P=0.001)$ to phenotypes determined by permutation tests $(n=10,000)$. Bar graphs (eh) show the phenotypic effects of $\mathrm{GK}$ and $\mathrm{BN}$ alleles at the chromosome 10 marker D10Got156, which was significantly

duodenal pancreas (data not shown). The effect in males alone was largely responsible for generating this QTL (Fig. 3e and f). Consistent linkages to IPCA and BCM in the duodenal pancreas were found in a region of chromosome 7 significantly linked to body weight in the cross (data not shown) and in a previous GKxBN F2 cross (Gauguier et al. 1996), but these effects were not statistically significant (maximum LOD 2.10 for IPCA) (Fig. 3c and d).

A highly significant QTL for plasma corticosterone concentration was detected on rat chromosome 1 (maximum LOD 4.2 at marker D1Got308) (Fig. 4a and Fig. 4b). This locus was denoted Pcort1. F2 animals that were homozygous for the GK allele or heterozygous at marker locus D1Got308 showed significantly reduced plasma corticosterone when compared to BN homozygous $\mathrm{F} 2$ rats at the same locus (Fig. 4c). This dominant effect of GK alleles at the locus on corticosterone levels was significant in both males and females.

No significant linkages to plasma GH, prolactin or IGF1 were found when data from the full cohort were analysed associated with BCM and IPCA in the splenic pancreatic region (spl) (e,f), and at the chromosome 7 marker D7Got88, which showed the strongest effects on BCM and IPCA in the duodenal pancreatic region (duo) (g, h). Phenotype means were calculated in males and females according to the genotype homozygous for the $\mathrm{BN}$ allele (black bar), heterozygous (hatched bar) or homozygous for the GK allele (white bar) at the loci. Number of rats carrying each genotype is shown in the bar chart. Results from the $F$-test and $P$-values are reported

(Supplementary Fig. S3). However, the single-sex genome scans indicated the existence of male-specific QTLs in the cross. The most marked differences were found for plasma GH and prolactin, which showed evidence of linkages (LOD > 4) in male rats to loci D6Got166 on chromosome $6(\mathrm{GH})$ and D17Rat34 on chromosome 17 (prolactin), whereas significance all but disappeared when considering females (data not shown). At both loci GK alleles were associated with increased concentrations of plasma $\mathrm{GH}$ and prolactin (Fig. S3e and Fig. S3f, Supplementary material) and showed a recessive effect on these variables.

\section{Discussion}

We have mapped genetic determinants of intrinsic islet phenotypes and circulating factors in the GK rat model of type 2 diabetes. The description of new QTLs linked to these variables provides new insights into the etiology of diabetes related phenotypes in this strain and underlines the 

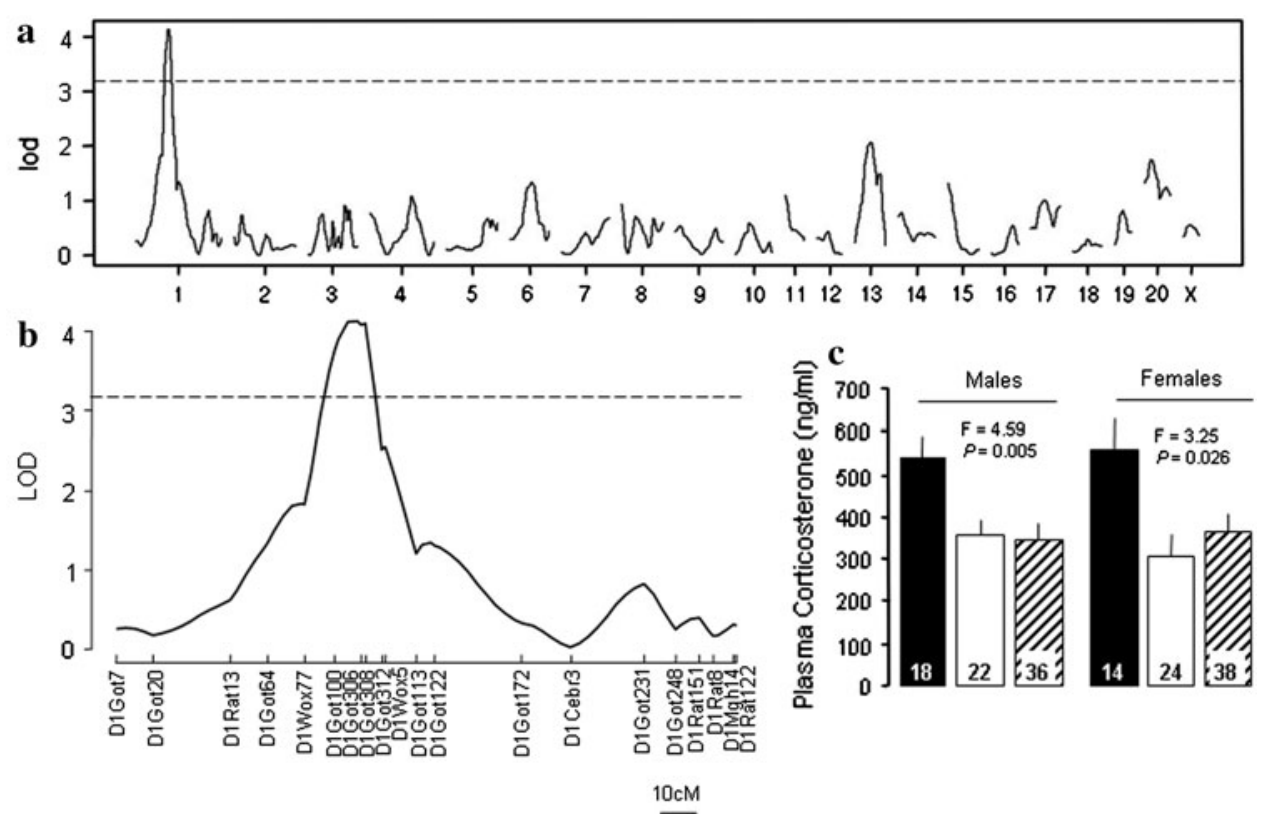

Fig. 4 Genetic control of plasma corticosterone concentration by the locus Pcort1 in the GKxBN F2 cross. LOD scores are plotted against markers mapped across the genome (a). Map distances calculated in the cross are reported in centimorgans (cM). Mapping details of the most significant QTL (LOD 4.2) in chromosome 1 are shown (b). Allelic effects on plasma corticosterone levels were calculated in

males and females according to the genotypes homozygous for the $\mathrm{BN}$ allele (black bar), heterozygous (hatched bar) or homozygous for the GK allele (white bar) at marker locus D1Got308 (c). Number of rats carrying each genotype is shown in the bar chart. Results from the $F$-test and $P$-values are reported

multiple and complex molecular mechanisms that collectively regulate islet function and have to be considered in diabetes genetic studies. These results complement previous studies that demonstrated anomalies in the genetic control of insulin secretion in this strain (Collins et al. 2006; Gauguier et al. 1996; Wallace et al. 2004; Wallis et al. 2004, 2008).

Genetic investigations into the causes of reduced beta cell mass are central to our understanding of molecular mechanisms which, in combination with insulin resistance, are involved in the onset and progression of type 2 diabetes (Kahn et al. 2009). However, screening phenotypes relevant to these clinical features represents a major challenge in human genetics. The GK strain, which exhibits both insulin resistance and structural and functional anomalies of the endocrine pancreas (Portha 2005), provides a powerful experimental system to establish an etiological basis for these phenotypes. Genetic studies in this strain have primarily focused on metabolic phenotypes (Dumas et al. 2007) and biological variables related to glucose regulation, lipid homeostasis (Argoud et al. 2006; Gauguier et al. 1996) and insulin secretion (Gauguier et al. 1996) in the same GK-BN strain combination. The QTL Bcml relevant to pancreas histology does not coincide with previously detected QTLs in the GK rat. Positional and functional candidate genes for $\mathrm{Bcml}$ include genes encoding $\mathrm{GH}$, signal transducers and activators of transcription (Stat3,
Stat5), which play central roles in mediating the biological effects of prolactin and GH in beta cells (Brelje et al. 2004; Mziaut et al. 2008), and the suppressor of cytokine signaling Socs-3, which inhibits GH-promoted beta-cell proliferation (Lindberg et al. 2005). In addition, microarray-based gene transcription profiling data showed that Stat3 is significantly overexpressed in GK (Hu et al. 2009).

Results from our genetic study showed a lack of correlation between islet morphological traits derived from distinct regions of the pancreas suggesting an apparent distinct genetic control of these phenotypes in the cross. The identification of genetic loci linked to histopathological phenotypes in duodenal and splenic regions, which largely correspond to pancreatic head and tail, respectively and have distinct embryological origins (Gittes 2009; Habener et al. 2005), may underlie mechanisms involved in the complex organogenesis of the endocrine pancreas. Even though beta cell mass is reduced in both pancreatic regions in type 2 diabetes (Rahier et al. 2008), density of islets and beta cells is very different in rodents, the tail of the pancreas containing higher number of islets and beta cells than the head (Rahier et al. 1983). The proportion of alpha-, delta- and PP-cells also differs in these regions. The QTL $B c m 1$ linked to beta cell mass in the splenic pancreas region may shed light on genes that cause an imbalance between apoptosis and replication/neogenesis in this islet rich region of the pancreas. Strongest linkage to islet 
phenotypes in the duodenal region of the pancreas was found at a locus of chromosome 7 significantly linked to body weight in this cross (LOD $>7$, data not shown) and in a previous GKxBN cross (Gauguier et al. 1996), suggesting a role of the underlying gene on islet growth. The identification of genes accounting for these islet histopathology phenotypes may provide new insights into molecular mechanisms that contribute to the maintenance of endocrine pancreas integrity.

To complement the genetic mapping of endocrine pancreas structural phenotypes, we investigated in the cross the genetic basis of the control of circulating factors known to have broad regulatory roles in islet function, development and survival, including IGF-1, prolactin, GH and corticosterone. Prolactin and GH are potent growth factors which up-regulate pancreatic beta-cell function and mass (BenJonathan et al. 2006; Zhang et al. 2009). They stimulate insulin gene transcription and secretion, increase beta-cell proliferation and inhibit beta-cell apoptosis (Ben-Jonathan et al. 2006; Brelje et al. 1993; Zhang et al. 2009). In contrast, glucocorticoids have opposing biological effects (Weinhaus et al. 2000). They inhibit beta-cell proliferation, reduce beta-cell viability, down regulate insulin production and secretion in vivo and in vitro (Lambillotte et al. 1997; Ranta et al. 2006) and contribute to insulin resistance (Andrews and Walker 1999). No significant linkage to plasma IGF-1 was found, and male-specific genetic effects on the control of plasma $\mathrm{GH}$ and prolactin concentrations were detected in chromosomes 6 and 17, respectively. The highly significant QTL Pcort1 linked to plasma corticosterone was detected in a region of rat chromosome 1 which maps $>30 \mathrm{cM}$ away from QTLs linked to glucose tolerance, adiposity, insulin secretion (Gauguier et al. 1996) and cholesterol metabolism (Argoud et al. 2006) in GKxBN F2 crosses. This QTL is distinct to the locus Scortl for serum corticosterone identified in an hypertensive rat strain, which maps to the telomeric end of chromosome 1 (Bilusic et al. 2004).

GK alleles are associated with increased concentrations of plasma GH and prolactin at both QTLs mapped to chromosomes 6 and 17, and reduced concentration of corticosterone at the QTL Pcortl. The effects of GK alleles would synergistically result in enhanced islet cell function and insulin secretion, and may compensate for the phenotypic consequences of GK alleles at other loci, including Bcml and QTLs linked to impaired glucose homeostasis. They are consistent with the role of GK alleles at previously described QTLs in GKxBN crosses which are associated with enhanced insulin secretion in vivo in response to arginine and glucose (Gauguier et al. 1996). The biological effects of GK alleles on increased beta cell mass and enhanced insulin secretion in vivo and in vitro have been demonstrated in a GK.BN congenic strain containing
GK haplotypes in a large region of chromosome 1 covering Pcort 1 and QTLs associated with insulin secretion, glucose tolerance, adiposity and plasma cholesterol (Wallis et al. 2008).

Genetic analysis of phenotypes measured in an F2 cohort combining female and male hybrids provided us with an opportunity to address sex-specific genetic control of islet morphological and hormonal phenotypes. As previously reported for the genetic control of glucose tolerance (Gauguier et al. 1996) and cholesterol metabolism (Argoud et al. 2006) in other GK $\times$ BN F2 cohorts, we identified significant or suggestive sex-specific loci linked to islet structural features and plasma hormones (BCM, IPCA, GH, prolactin) that are mostly due to an effect in males. We also noted female specific dominant effects of GK alleles on islet structural phenotypes in the duodenal pancreatic region. Sexual dimorphism is a hallmark of genetic studies of complex traits (Weiss et al. 2006). It may implicate sex-specific responsiveness to environmental factors and interactions between autosomal QTLs and chromosome $\mathrm{X}$ loci that congenic strains, where alleles in this chromosome do no segregate (Collins et al. 2006), can help investigate.

Collectively, results from our genetic studies suggest an involvement of genes causing islet structural and functional changes in the etiology of diabetes in the GK rat. Even though the GK strain was produced through repeated breeding of rats selected on the sole criterion of glucose intolerance (Goto et al. 1988), suggesting the eventual isolation of natural variants that predominantly contribute to impaired glucose homeostasis, we have been able to identify gene loci involved in islet structure and function. These gene variants may have randomly aggregated in the GK during the process of selective breeding or may have been isolated to either compensate or further deteriorate insulin resistance. The intricate roles of insulin resistance and beta cell dysfunction in diabetes pathogenesis is illustrated by the observation that partial pancreatectomy has little impact on glucose homeostasis unless glucose intolerance or preexisting beta cell dysfunction are present (Menge et al. 2009; Slezak and Andersen 2001).

Results from this study provide novel insights into etiological factors that contribute to diabetes in the GK and complement previous genetic studies in this strain which primarily focused on glucose tolerance, insulin secretion and metabolic phenotypes. Genetic investigations in the GK suggest a predominant role of insulin resistance in the etiology of diabetes in this strain that can be further investigated in combination with islet morphological phenotypes in congenic strains to fine map the underlying genes and characterise their function (Collins et al. 2006; Wallis et al. 2008). There are important differences in islet architecture and cellular composition between humans and 
rodents (Steiner et al. 2010) that may hamper translational studies of rodent genetic and physiological data in humans. Genetic studies dissecting diabetes related variables in a rodent model of spontaneous type 2 diabetes demonstrate the complex phenotypic consequences of naturally occurring variants which collectively contribute to the diabetic phenotype.

Acknowledgments This work was supported by the Wellcome Trust and grants from the European Commission FGENTCARD (Functional genomic diagnostic tools for coronary artery disease, LSHG-CT-2006-037683). DG holds a Wellcome Senior Fellowship in Basic Biomedical Science (057733). SPW is a recipient of a Wellcome Prize Studentship in Bioinformatics and Statistical Genetics.

Open Access This article is distributed under the terms of the Creative Commons Attribution Noncommercial License which permits any noncommercial use, distribution, and reproduction in any medium, provided the original author(s) and source are credited.

\section{References}

Andrews RC, Walker BR (1999) Glucocorticoids and insulin resistance: old hormones, new targets. Clin Sci (Lond) 96:513-523

Argoud K, Wilder SP, McAteer MA, Bihoreau MT, Ouali F, Woon PY, Wallis RH, Ktorza A, Gauguier D (2006) Genetic control of plasma lipid levels in a cross derived from normoglycaemic Brown Norway and spontaneously diabetic Goto-Kakizaki rats. Diabetologia 49:2679-2688

Ben-Jonathan N, Hugo ER, Brandebourg TD, LaPensee CR (2006) Focus on prolactin as a metabolic hormone. Trends Endocrinol Metab 17:110-116

Bihoreau MT, Gauguier D, Kato N, Hyne G, Lindpaintner K, Rapp JP, James MR, Lathrop GM (1997) A linkage map of the rat genome derived from three F2 crosses. Genome Res 7:434-440

Bilusic M, Bataillard A, Tschannen MR, Gao L, Barreto NE, Vincent M, Wang T, Jacob HJ, Sassard J, Kwitek AE (2004) Mapping the genetic determinants of hypertension, metabolic diseases, and related phenotypes in the lyon hypertensive rat. Hypertension 44:695-701

Brelje TC, Scharp DW, Lacy PE, Ogren L, Talamantes F, Robertson M, Friesen HG, Sorenson RL (1993) Effect of homologous placental lactogens, prolactins, and growth hormones on islet Bcell division and insulin secretion in rat, mouse, and human islets: implication for placental lactogen regulation of islet function during pregnancy. Endocrinology 132:879-887

Brelje TC, Stout LE, Bhagroo NV, Sorenson RL (2004) Distinctive roles for prolactin and growth hormone in the activation of signal transducer and activator of transcription 5 in pancreatic islets of langerhans. Endocrinology 145:4162-4175

Broman KW, Wu H, Sen S, Churchill GA (2003) R/qtl: QTL mapping in experimental crosses. Bioinformatics 19:889-890

Collins SC, Wallis RH, Wilder SP, Wallace KJ, Argoud K, Kaisaki PJ, Bihoreau MT, Gauguier D (2006) Mapping diabetes QTL in an intercross derived from a congenic strain of the Brown Norway and Goto-Kakizaki rats. Mamm Genome 17:538-547

Doerge RW, Churchill GA (1996) Permutation tests for multiple loci affecting a quantitative character. Genetics 142:285-294
Dumas ME, Wilder SP, Bihoreau MT, Barton RH, Fearnside JF, Argoud K, D'Amato L, Wallis RH, Blancher C, Keun HC, Baunsgaard D, Scott J, Sidelmann UG, Nicholson JK, Gauguier D (2007) Direct quantitative trait locus mapping of mammalian metabolic phenotypes in diabetic and normoglycemic rat models. Nat Genet 39:666-672

Edlund H (2001) Developmental biology of the pancreas. Diabetes 50(Suppl 1):S5-S9

Gauguier D, Froguel P, Parent V, Bernard C, Bihoreau MT, Portha B, James MR, Penicaud L, Lathrop M, Ktorza A (1996) Chromosomal mapping of genetic loci associated with non-insulin dependent diabetes in the GK rat. Nat Genet 12:38-43

Gittes GK (2009) Developmental biology of the pancreas: a comprehensive review. Develop Biol 326:4-35

Goto Y, Suzuki KI, Sasaki M, Ono T, Abe S (1988) GK rat as a model of nonobese, noninsulin-dependent diabetes. Selective breeding over 35 generations. In: Shafrir E, Renold AE (eds) Lessons from animal diabetes II. Libbey, J, London, pp 301-303

Habener JF, Kemp DM, Thomas MK (2005) Minireview: transcriptional regulation in pancreatic development. Endocrinology 146:1025-1034

Haley CS, Knott SA (1992) A simple regression method for mapping quantitative trait loci in line crosses using flanking markers. Heredity 69:315-324

Hu Y, Kaisaki PJ, Argoud K, Wilder SP, Wallace KJ, Woon PY, Blancher C, Tarnow L, Groop PH, Hadjadj S, Marre M, Parving HH, Farrall M, Cox RD, Lathrop M, Vionnet N, Bihoreau MT, Gauguier D (2009) Functional annotations of diabetes nephropathy susceptibility loci through analysis of genome-wide renal gene expression in rat models of diabetes mellitus. BMC Med Genomics 2:41

Ihaka R, Gentleman R (1996) R: a language for data analysis and graphics. J Comput Graph Statist 5:299-314

Kahn SE (2003) The relative contributions of insulin resistance and beta-cell dysfunction to the pathophysiology of Type 2 diabetes. Diabetologia 46:3-19

Kahn SE, Zraika S, Utzschneider KM, Hull RL (2009) The beta cell lesion in type 2 diabetes: there has to be a primary functional abnormality. Diabetologia 52:1003-1012

Kargar C, Ktorza A (2008) Anatomical versus functional beta-cell mass in experimental diabetes. Diabetes Obes Metab 10(Suppl 4):43-53

Lambillotte C, Gilon P, Henquin JC (1997) Direct glucocorticoid inhibition of insulin secretion. An in vitro study of dexamethasone effects in mouse islets. J Clin Invest 99:414-423

Lindberg K, Ronn SG, Tornehave D, Richter H, Hansen JA, Romer J, Jackerott M, Billestrup N (2005) Regulation of pancreatic betacell mass and proliferation by SOCS-3. J Mol Endocrinol 35:231-243

Menge BA, Schrader H, Breuer TG, Dabrowski Y, Uhl W, Schmidt WE, Meier JJ (2009) Metabolic consequences of a 50\% partial pancreatectomy in humans. Diabetologia 52:306-317

Mziaut H, Kersting S, Knoch KP, Fan WH, Trajkovski M, Erdmann K, Bergert H, Ehehalt F, Saeger HD, Solimena M (2008) ICA512 signaling enhances pancreatic beta-cell proliferation by regulating cyclins D through STATs. Proc Natl Acad Sci USA 105:674-679

Portha B (2005) Programmed disorders of beta-cell development and function as one cause for type 2 diabetes? The GK rat paradigm. Diabetes Metab Res Rev 21:495-504

Puri S, Hebrok M (2010) Cellular plasticity within the pancreaslessons learned from development. Develop Cell 18:342-356

Rahier J, Goebbels RM, Henquin JC (1983) Cellular composition of the human diabetic pancreas. Diabetologia 24:366-371

Rahier J, Guiot Y, Goebbels RM, Sempoux C, Henquin JC (2008) Pancreatic beta-cell mass in European subjects with type 2 diabetes. Diabetes Obes Metab 10(Suppl 4):32-42 
Ranta F, Avram D, Berchtold S, Dufer M, Drews G, Lang F, Ullrich S (2006) Dexamethasone induces cell death in insulin-secreting cells, an effect reversed by exendin-4. Diabetes 55:1380-1390

Slezak LA, Andersen DK (2001) Pancreatic resection: effects on glucose metabolism. World J Surg 25:452-460

Spooner BS, Walther BT, Rutter WJ (1970) The development of the dorsal and ventral mammalian pancreas in vivo and in vitro. J Cell Biol 47:235-246

Stam P (1993) Construction of integrated genetic linkage maps by means of a new computer package. JoinMap. Plant J 3:739-744

Steiner DJ, Kim A, Miller K, Hara M (2010) Pancreatic islet plasticity: interspecies comparison of islet architecture and composition. Islets 2:135-145

Ury HK (1976) A comparison of four procedures for multiple comparisons among means (pairwise contrasts) for arbitrary samples sizes. Technometrics 18:89-97

Wallace KJ, Wallis RH, Collins SC, Argoud K, Kaisaki PJ, Ktorza A, Woon PY, Bihoreau MT, Gauguier D (2004) Quantitative trait locus dissection in congenic strains of the Goto-Kakizaki rat identifies a region conserved with diabetes loci in human chromosome 1q. Physiol Genomics 19:1-10

Wallis RH, Wallace KJ, Collins SC, McAteer M, Argoud K, Bihoreau MT, Kaisaki PJ, Gauguier D (2004) Enhanced insulin secretion and cholesterol metabolism in congenic strains of the spontaneously diabetic (Type 2) Goto Kakizaki rat are controlled by independent genetic loci in rat chromosome 8. Diabetologia 47:1096-1106

Wallis RH, Collins SC, Kaisaki PJ, Argoud K, Wilder SP, Wallace KJ, Ria M, Ktorza A, Rorsman P, Bihoreau MT, Gauguier D (2008) Pathophysiological, genetic and gene expression features of a novel rodent model of the cardio-metabolic syndrome. PLoS One 3:e2962

Weinhaus AJ, Bhagroo NV, Brelje TC, Sorenson RL (2000) Dexamethasone counteracts the effect of prolactin on islet function: implications for islet regulation in late pregnancy. Endocrinology 141:1384-1393

Weiss LA, Pan L, Abney M, Ober C (2006) The sex-specific genetic architecture of quantitative traits in humans. Nat Genet $38: 218-222$

Wilder SP, Bihoreau MT, Argoud K, Watanabe TK, Lathrop M, Gauguier D (2004) Integration of the rat recombination and EST maps in the rat genomic sequence and comparative mapping analysis with the mouse genome. Genome Res 14:758-765

Zhang F, Sjoholm A, Zhang Q (2009) Growth hormone signaling in pancreatic beta-cells - calcium handling regulated by growth hormone. Mol Cell Endocrinol 297:50-57 